\title{
Risk Factors for Ischaemic Stroke in an Omani Community A case-control study
}

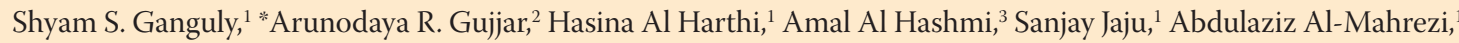
Abdullah R. Al-Asmi²

\begin{abstract}
Objectives: Stroke is recognised as the third most common cause of mortality and it has an increasing incidence in developing countries. Recognition and control of risk factors are of prime importance in the prevention of stroke. This study aimed to examine the characteristics of ischaemic stroke (IS) patients in Oman and quantify its various risk factors using a case-control model. Methods: This case-control study was conducted from January 2012 to March 2013 at Sultan Qaboos University Hospital and Royal Hospital, Muscat. Adult Omani patients with IS who were admitted to either hospital were compared to age- and gender-matched controls. Demographic factors and frequency of various conventional risk factors were documented. Univariate and stepwise multivariate logistic regression analyses were performed to evaluate the risk factors associated with IS. Results: A total of 255 patients and age-and gender-matched controls were included in this study. The mean age was $62.2 \pm 13.2$ years and $63.14 \%$ were male. Most cases (89.02\%) were above 45 years of age. Cardio-embolism (31.76\%) was the commonest mechanism of IS. Stepwise multiple logistic regression model revealed that family history of stroke was the strongest independent risk factor, followed by hypertension and high-density lipoprotein levels (odds ratio: 10.10, 5.17 and 3.34, respectively; $P<0.01$ each). Conclusion: Cardio-embolism was the predominant mechanism of IS in this study. Family history of stroke, hypertension and reduced high-density lipoprotein were the leading independent risk factors. Strong emphasis on screening for risk factors, control of hypertension and lifestyle modification for those with a family history of stroke would be expected to emerge as the major stroke-preventive measures in Oman.
\end{abstract}

Keywords: Ischemic Stroke; Risk Factors; Case-Control Study; Oman.

\section{AdVANCES IN KNOWLEDGE}

In the context of the rising prevalence of stroke in many developing countries, recognition of the predominant risk factors in a community is of prime importance. In this study, cardio-embolism was observed to be the predominant cause of ischaemic stroke (IS) in Omani subjects and a family history of stroke, hypertension and dyslipidemia were the most prevalent risk factors.

\section{Applications to Patient Care}

IS in Omani population shows a significant association with a family history of stroke and hypertension, which are predominant risk factors.

Since cardio-embolism is a major mechanism of IS in Oman, investigations to recognise cardiac dysrhythmias and valvular heart diseases should be a routine part of stroke evaluation.

Preventive measures aimed at reducing the incidence of IS in Oman should focus on modifiable risk factors such as hypertension, dyslipidaemia and lifestyle modification (e.g. control of smoking and increasing physical activity).

$\mathrm{T}$ HE GLObal burden of STROKE IS INCREasing despite the declining stroke mortality rates. A more comprehensive approach to primary prevention of stroke, which targets individuals at all levels of risk and is integrated with prevention strategies for other diseases that share common risk factors, is required. ${ }^{1}$ Ischaemic stroke (IS) is a heterogeneous multifactorial disorder that is identified by the sudden onset of neurologic deficits secondary to vascular obstruction and its varied manifestations are determined by the location of injury in the brain. While the burden of IS is gradually reducing in several developed nations, most developing countries have an increasing incidence. The high domestic and global burden of IS makes it an important public health concern. ${ }^{2}$

Stroke-related deaths in Oman contribute to approximately $6.58 \%$ of the total deaths, with the age-adjusted mortality rate being 39.08 per 100,000 . It is the third leading cause of mortality in Oman after coronary heart disease and diabetes mellitus. ${ }^{3}$ Oman, similar to the Middle Eastern population, has a relatively higher load of conditions that predispose one to cardiovascular disease - hypertension, obesity, diabetes mellitus and dyslipidemia. ${ }^{4}$ Preliminary observations from hospital-based stroke registries in Oman as well as in the Gulf Cooperation Council (GCC) countries suggest that stroke occurs about 
a decade earlier in the Omani population when compared to Western cohorts. ${ }^{5,6}$ In this context, it is important to examine the actual risk factors of stroke in this population in order to identify the preventive measures that might need to be pursued with a greater emphasis for the prevention of stroke. To the best of the authors' knowledge, no study has addressed the prevalence of IS or a formal quantification of risk factors associated with IS in Oman. This hospitalbased study aimed to study the characteristics of IS as well as to investigate and quantify the risk factors associated with IS among Omani patients using a case-control design.

\section{Methods}

This prospective, hospital-based case-control study was conducted from January 2012 to March 2013 on Omani patients with IS who were admitted to two premier hospitals in Oman - Sultan Qaboos University Hospital (SQUH) and Royal Hospital, Muscat. Both the hospitals serve as national referral centres for specialised treatment of all types of stroke from all over Oman and have similar referral patterns. Participants aged $\geq 18$ years who were newly diagnosed with IS and admitted to the hospital were recruited as 'cases'. IS was defined as the occurrence of acute focal neurologic deficits due to a vascular insult as confirmed by clinical examination and brain computed tomography and/ or magnetic resonance imaging. ${ }^{7}$ IS subtypes were defined according to the Trial of Org 10172 in Acute Stroke Treatment (TOAST) criteria. ${ }^{8}$ This is a widely used classification with good reliability and high sensitivity and specificity. ${ }^{9}$ Five subtypes of IS were recognised: large-artery atherosclerosis, small-vessel disease or lacunar stroke, cardio-embolic stroke (CE), stroke of other defined aetiologies or stroke of other undetermined aetiology. ${ }^{8,10}$ Conditions that mimic IS, such as intracerebral haemorrhage, cerebral venous sinus thrombosis, brain tumours and a demyelinating condition, were excluded.

The control subjects included patients admitted to the same hospitals with non-medical conditions or for elective non-neurologic surgeries such as hernia and cataracts. None of the controls had a history of stroke according to a detailed documented medical evaluation. The controls were matched for age $( \pm 2$ years) and gender.

The information collected from cases included demographic characteristics, IS subtypes, risk factors, medical comorbidities, laboratory tests, duration of hospital admission and outcomes such as death, survived or left against medical advice. Face-to-face interviews of admitted subjects or knowledgeable
Table 1: Baseline and clinical characteristics of ischemic stroke patients in Oman $(\mathrm{N}=255)$

$\begin{array}{lc}\text { Characteristic } & \mathbf{n}(\%) \\ \text { Mean age in years SD } & 62.2 \pm 13.2 \\ \text { Gender } & \\ \text { Male } & 161(63.14) \\ \text { Female } & 94(34.86) \\ \text { Age group in years } & \\ \text { 18-45 } & 28(10.98) \\ 46-65 & 117(45.88) \\ >65 & 110(43.14) \\ \text { Ischaemic stroke sub-type } & \\ \text { LAA } & 72(28.24) \\ \text { CE } & 81(31.76) \\ \text { LS } & 61(23.92) \\ \text { OD } & 6(2.35) \\ \text { UD } & 35(13.73) \\ \text { Mortality } & \\ \text { In hospital prior to discharge } & 8(3.14) \\ \text { Documented within three months post- } \\ \text { Tischarge }\end{array}$

$L A A=$ large artery atherosclerosis; $C E=$ cardio-embolism; $L S=$ lacunar stroke; $O D=$ other determined cause; $U D=$ undetermined cause; $T I A=$ transient ischaemic attack.

relatives (or telephonic interviews as required) helped to complete any missing information. Information obtained from control subjects included demographics and the presence of risk factors.

Sample sizes for the cases and controls were estimated using the method suggested by Schlesselman. ${ }^{11}$ This study considered the prevalence of hypertension as prime exposure rate in controls as $20 \%$ as well as the risk of developing stroke in exposed subjects with an odds ratio (OR) of 2.0 with 95\% confidence interval (CI) and $80 \%$ power; the sample sizes for the cases and controls were estimated at 255 each.

The risk factors of IS included hypertension, diabetes mellitus, dyslipidaemia, cardiac diseases, family history, smoking, alcohol, physical activity and obesity. Conventional definitions of risk factors were used in this study. ${ }^{8,12,13}$ They can be briefly described as follows: (1) hypertension was defined as systolic blood pressure of $\geq 140 \mathrm{mmHg}$ and/or diastolic blood pressure of $\geq 90 \mathrm{mmHg}$ or current use of antihypertensive 
Table 2: Prevalence of risk factors of ischaemic stroke and their crude odds ratios among cases of ischaemic stroke and controls in Oman $(\mathrm{N}=255$ each)

\begin{tabular}{|c|c|c|c|c|}
\hline \multirow[t]{2}{*}{ Risk factor } & \multicolumn{2}{|c|}{ n (\%) } & \multirow[t]{2}{*}{ OR $(95 \% \mathrm{CI})$} & \multirow[t]{2}{*}{$P$ value } \\
\hline & Cases & Controls & & \\
\hline Hypertension & $202(79.22)$ & $108(42.35)$ & $5.18(3.50-7.67)$ & $<0.001$ \\
\hline Diabetes mellitus & $159(62.35)$ & $114(44.7)$ & $2.05(1.44-2.92)$ & $<0.001$ \\
\hline Dyslipidaemia & $173(67.84)$ & $111(43.5)$ & $2.73(1.91-3.93)$ & $<0.001$ \\
\hline Chronic kidney disease & $16(6.27)$ & $13(5.1)$ & $1.24(0.58-2.64)$ & 0.566 \\
\hline Heart disease & $67(26.27)$ & $30(11.8)$ & $2.67(1.66-4.24)$ & $<0.001$ \\
\hline Smoking $(\mathrm{n}=210)$ & $69(27.06)$ & $43(16.86)$ & $1.84(1.18-2.87)$ & 0.006 \\
\hline No regular exercise $(\mathrm{n}=204)$ & $123(48.24)$ & $76(9.80)$ & $2.54(1.70-3.78)$ & $<0.001$ \\
\hline Alcohol consumption $(\mathrm{n}=205)$ & $30(11.76)$ & $15(5.88)$ & $2.17(1.13-4.17)$ & 0.020 \\
\hline BMI of $\geq 30$ in $\mathrm{kg} / \mathrm{m} 2(\mathrm{n}=107)$ & $40(15.69)$ & $55(21.57)$ & $1.32(0.80-2.19)$ & 0.275 \\
\hline Family history of stroke (first-degree relatives) $(n=201)$ & $45(17.65)$ & $7(2.75)$ & $8.08(3.54-18.41)$ & $<0.001$ \\
\hline $\mathrm{TC}$ of $\geq 5.2 \mathrm{in} \mathrm{mmol} / \mathrm{L}(\mathrm{n}=226)$ & $108(42.35)$ & $80(31.37)$ & $1.68(1.15-2.45)$ & 0.007 \\
\hline $\mathrm{HDL}-\mathrm{C}$ of $<1.03 \mathrm{in} \mathrm{mmol} / \mathrm{L}(\mathrm{n}=225)$ & $119(46.67)$ & $59(23.14)$ & $3.18(2.14-4.72)$ & $<0.001$ \\
\hline $\mathrm{LDL}-\mathrm{C}$ of $\geq 3.4$ in $\mathrm{mmol} / \mathrm{L}(\mathrm{n}=223)$ & $112(43.92)$ & $72(28.24)$ & $2.12(1.44-3.11)$ & $<0.001$ \\
\hline $\mathrm{TG}$ of $\geq 1.7 \mathrm{in} \mathrm{mmol} / \mathrm{L}(\mathrm{n}=225)$ & $50(19.61)$ & $41(16.08)$ & $1.29(0.81-2.05)$ & 0.281 \\
\hline
\end{tabular}

$O R=$ odds ratio; $C I=$ confidence interval; $B M I=$ body mass index; $T C=$ total cholesterol; $H D L-C=$ high-density lipoprotein cholesterol; $L D L-C=$ lowdensity lipoprotein cholesterol; $T G$ = triglycerides.

medicines; (2) individuals who were on anti-diabetic drugs or had a fasting blood glucose of $\geq 7.0 \mathrm{mmol} / \mathrm{L}$ or a glycated haemoglobin of $>6.5$ were considered to have diabetes mellitus; (3) dyslipidaemia was defined as the presence of one or more of the following: (a) total cholesterol (TC) $\geq 5.2 \mathrm{mmol} / \mathrm{L}$; (b) high-density lipoprotein cholesterol (HDL-C) $<1.03 \mathrm{mmol} / \mathrm{L}$; (c) low-density lipoprotein cholesterol (LDL-C) $\geq 3.4$ $\mathrm{mmol} / \mathrm{L}$; or (d) triglycerides (TG) $\geq 1.7 \mathrm{mmol} / \mathrm{L}$; (4) cardiac disease was defined as the presence (in the past or currently) of a cardiac condition such as atrial fibrillation, ischaemic heart disease, cardiomyopathy or valvular heart disease, which is considered a risk factor for IS; (5) family history of IS was recognised when the subject had a first-degree relative (maternal, paternal or sibling) with a diagnosis of stroke; (6) individuals who were not doing regular exercise for at least 20 minutes three times a week were considered as lacking physical exercise; (7) body mass index (BMI) was calculated as weight/height2 $\left(\mathrm{kg} / \mathrm{m}^{2}\right)$ and being overweight meant having a BMI $\geq 30 \mathrm{~kg} / \mathrm{m}^{2}$; and (8) a history of smoking and alcohol consumption history were classified as binary exposure variables based on any use currently or in the past versus never having smoked or consumed alcohol.

Continuous variables were expressed as means and standard deviations; categorical variables were expressed as numbers and percentages. Significant differences between cases and controls for various categorical variables were explored using a Chi-square test. The relationships between the different risk factors and the development of stroke were estimated using univariate analysis and expressed in terms of crude ORs with their 95\% CIs. A multivariable logistic regression model was fitted to investigate the association between the significant factors found under the univariate analysis; the adjusted ORs with their 95\% CIs were obtained using a backward conditional multiple logistic regression analysis. The analyses were carried out using Statistical Package for the Social Sciences (SPSS), Version 23 (IBM Corp., Armonk, New York, USA). ${ }^{14}$ A $P$ value of $<0.05$ (2-tailed tests) was considered statistically significant.

The Medical Research and Ethics Committee of the College of Medicine and Health Sciences, Sultan Qaboos University approved the study. All participants provided their written informed consent prior to participating in the study.

\section{Results}

The ratio of males to females among cases was 1.7:1. Their ages ranged from 22 to 90 years with a mean of $62.2 \pm 13.2$ years. Male and female patients were comparable in their mean ages $(P=0.54)$. A majority of the cases $(89.02 \%)$ were in the age group 
Table 3: Results of multiple logistic regression analysis exploring the relationship of ischaemic stroke risk factors in Oman

$\begin{array}{lcc}\text { Factor } & \text { OR }(\mathbf{9 5} \% \mathbf{C I}) & \boldsymbol{P} \text { value } \\ \text { Family history } & 10.10(3.41-29.98) & <0.001 \\ \text { Hypertension } & 5.17(2.92-9.14) & <0.001 \\ \text { No regular exercise } & 2.65(1.56-4.50) & <0.001 \\ \text { Alcohol consumption } & 2.28(0.88-5.91) & 0.090 \\ \text { HDL-C } & 3.34(1.93-5.78) & <0.001 \\ \text { LDL-C } & 2.96(1.72-5.09) & <0.001\end{array}$

$O R=$ odds ratio; $C I=$ confidence interval; $H D L-C=$ high-density lipoprotein cholesterol; $L D L-C=$ low-density lipoprotein cholesterol.

above 45 years. A total of 234 (91.76\%) patients were recruited from Royal Hospital and 21 (8.24\%) from SQUH. Among patients with IS, cardio-embolism was the commonest mechanism (31.76\%) followed by large artery atherosclerosis (28.24\%) [Table 1]. On subgroup analysis, cardio embolic stroke was found to be the predominant subtype among those $<65$ years old (34.50\%), followed by stroke due to small artery occlusion in $26.20 \%$ of subjects. In contrast, among older patients, large artery atherosclerosis was the main subtype, accounting for $37.30 \%$ of the cases. Among the cases of IS, $14.51 \%$ had a history of transient ischaemic attack (TIA). The prevalence of TIA was $15.00 \%$ among males and $16.70 \%$ among females with no significant difference $(P=0.73)$. Almost one-third of the patients (35.69\%) had a previous history of one or more episodes of stroke.

Family history of stroke in first-degree relatives was the strongest independent risk factor with an OR of 8.08 (95\% CI: 3.54-18.41) followed by hypertension with an OR of 5.18 (95\% CI: 3.50-7.67). Decreased levels of HDL-C (OR = 3.18; 95\% CI: 2.14-4.72) and higher LDL-C $(\mathrm{OR}=2.12$; 95\% CI: $1.44-3.11)$ and TC levels $(\mathrm{OR}=1.68$; 95\% CI: $1.15-2.45)$ were noted among the cases when compared to the controls $(P<0.01$ each). However, the level of TG was not significantly associated with IS $(P=0.28)$. Other risk factors, namely ischaemic heart disease $(\mathrm{OR}=2.67$; 95\% CI: 1.66-4.24) and diabetes mellitus $(\mathrm{OR}=2.05$; 95\% CI: 1.44-2.92) were also found to be associated with IS. Among lifestyle practices, lack of physical exercise $(\mathrm{OR}=2.54 ; 95 \% \mathrm{CI}: 1.70-3.78)$, history of alcohol consumption $(\mathrm{OR}=2.17$; 95\% CI: 1.13-4.17) and history of smoking (OR $=1.84$; 95\% CI: 1.18-2.87) were observed to be more prevalent among the cases when compared to the control subjects ( $P<0.05$ each) [Table 2].

Estimation of adjusted ORs based on stepwise multiple logistic regression analysis reconfirmed that a family history of stroke $(\mathrm{OR}=10.10$; 95\% CI: 3.41-29.98) was the strongest independent risk factor followed by hypertension (OR $=5.17$; 95\% CI: $2.92-$ 9.14) and HDL-C (OR = 3.34; 95\% CI: 1.93-5.78; $P$ $<0.01$ each) [Table 3].

\section{Discussion}

Stroke is a common non-communicable disease that has a variable profile of risk factors among different communities. The use of a case-control model, as in the current study, is a valid method of exploring the risk factors of stroke and provided an opportunity to quantify the burden of the various risk factors present in patients with stroke compared to controls in terms of OR; it is also an inexpensive and easier approach. In contrast, a cross-sectional study would have entailed the study of a larger number of patients but would have provided prevalence measures though with poorer differentiation of the cause-effect relationship.

Several of the observations in this study were comparable to those present in the recently published literature on IS within the region. The mean age of IS cases in the current study was similar to that observed in the Outcomes in Patients with TIA and Cerebrovascular disease (OPTIC) registry (64.1 \pm 10.4 years) and the Prevention of cerebrovascular and cardiovascular Events of ischaemic origin with teRutroban in patients with a history oF ischaemic strOke or tRansient ischaeMic attack (PERFORM) study. ${ }^{14}$ Although IS due to cardio embolism was more prevalent in the current study compared to the rates reported in other Arab studies, the prevalence of heart disease and atrial fibrillation were found to be similar. ${ }^{6,11}$ Large artery stroke is often the more prevalent subtype; however, it had a slightly lower prevalence than CE in the current study. 5 ,6 The most likely reason for this discrepancy may have been sampling bias. Most patients with IS underwent tests for the identification of a cardio-embolic source (viz. Holter study and echocardiography). Data also revealed that cardio-embolism had the worst outcomes among IS and was associated with a higher fatality. This was also recognised in several earlier studies and this is mainly related to its pathophysiology. ${ }^{15-17}$ Specifically, cardioembolic stroke is often associated with relatively larger infarctions consequent to major vessel occlusion by the thrombus. ${ }^{18}$

Modifiable risk factors play an important role in the development of stroke. ${ }^{19}$ The prevalence of different risk factors of IS varies among populations. The overall prevalence of risk factors in the current study appeared to be similar to those reported by a 
stroke registry cohort from Oman as well as in the GCC region. ${ }^{5,6}$ In agreement with the GCC registry, the current study showed that hypertension and dyslipidaemia had a higher prevalence in IS compared to controls. ${ }^{6}$ Hypertension was nearly two times more prevalent among IS patients when compared to controls. This exceeds the range reported by other Arab countries but is comparable to stroke patients from other Asian populations such as Pakistan. ${ }^{20}$ In contrast, though diabetes mellitus was observed to be significantly associated with IS on conducting a univariate analysis, it did not emerge as a significant risk factor on conducting a multivariate analysis in this study. A possible reason for this was the overall higher prevalence of diabetes mellitus in the control population as well. This observation could be supported by reports of a relatively high prevalence of diabetes mellitus among adults in Oman. ${ }^{21}$

In the current study, the strongest risk factor for IS was observed to be a family history of stroke in first degree relatives, with a proportion nearly similar to that observed in studies from the United Arab Emirates (20\%) and Saudi Arabia (14.1\%). ${ }^{22}$ The relatively higher proportion of consanguinity in Oman may explain this observation..$^{23}$ This observation may have several implications. The relatively high familial burden of stroke noted in this study may indeed point to specific genetic factors underpinning atherosclerotic disease in the Omani population. Other possibilities are a stronger genetic predisposition to vascular risk factors such as hypertension or dyslipidaemias, indirectly influencing stroke incidence or, rarely, the prevalence of one or more specific single-gene mutations that are associated with stroke. This observation also suggested that studies utilising a genome-wide analysis involving a significantly large number of families with stroke burden may have a reasonable chance of recognising such factors. Indeed, a few large and well-powered genome-wide association study analyses have uncovered significant associations in IS. ${ }^{2}$ The findings of a prospective hospital-based registry that collected data from four Arabian Gulf countries also supports the concept that IS is a poly-aetiologic disease and that race/ethnic differences and genetic factors may be the reasons for the differences between Arab and Western populations. ${ }^{6}$ An effort towards exploring the genetic basis of IS has been initiated at our centre in the form of genetic analysis of apolipoprotein profile using a case-control design. An ongoing stroke registry also aims to include genetic studies.

Many factors ranging from differences in physical activity and nutrition patterns to genetic predisposition are likely to contribute to the higher prevalence of diabetes and obesity in this region. ${ }^{24,25}$ The large, multinational OPTIC Registry and PERFORM Trial observed the trend of a higher frequency of conventional risk factors for stroke as well as inadequately managed diabetes mellitus, hypertension and tobacco smoking in patients from Arab countries. ${ }^{14}$ Though a significantly higher prevalence of diabetes mellitus among IS cases was noted in this study, diabetes mellitus and smoking were not found to be important risk factors on conducting a logistic regression analysis. Earlier studies have observed a younger age of occurrence of stroke in Arab countries. ${ }^{14}$ While $89.02 \%$ of the cases in the present study were in the age group above 45 years, the mean age of IS patients was at least a decade less than that reported in Western populations. A larger study may be required to provide a plausible explanation for these findings related to diabetes, smoking and younger age among the cases in this study.

This study had several limitations. A relatively small number of patients were sourced from two major health care institutions in the country, both located in the same city; this may limit the generalisability of the observations to more distant communities of the country. However, as both institutions serve the same population and have similar referral practices, they were unlikely to recruit different patterns of cases for the study. Familial occurrence of stroke could not be explored in detail with regard to patterns of inheritance, relation to risk factors, etc. In addition, patients with TIA who were discharged early for outpatient follow-up could not be objectively assessed. While heart disease (inclusive of different conditions) as a risk factor was studied, certain risk factors such as atrial fibrillation or other specific heart diseases were not addressed in this study. Further well-designed comparative studies of Arabian populations including other ethnic communities are required to explore these concepts.

\section{Conclusion}

In the Omani population, IS was found to have a significant association with a family history of stroke and hypertension, which are the predominant risk factors. Since cardio embolism is a major mechanism of IS in Oman, investigations to recognise cardiac dysrhythmias and valvular heart diseases should be a routine part of stroke evaluation. Preventive measures aimed at reducing the incidence of IS in Oman should focus on modifiable risk factors such as hypertension, dyslipidaemia and lifestyle modification (e.g. for control of smoking, improving physical activity, etc.). Further studies that explore the familial burden of stroke in Oman with the help of modern genetic 
studies may provide an insight into possible genebased interventions for the prevention of stroke as well as other related non-communicable disorders.

\section{CONFLICT OF INTEREST}

The authors declare no conflicts of interest.

\section{FUNDING}

This study was supported by an internal grant (IG/ MED/FAMCO/13/01) at Sultan Qaboos University.

\section{AUTHORS' CONTRIBUTION}

SSG and ARG conceptualised and designed the study. SSG, ARG, AH, AM and ARA supervised the work. $\mathrm{HH}$ developed the proposal and SSG acquired the ethical approval and funding. ARG, HH, AH and ARA collected the data, while SSG and SJ analysed the data. SSG, ARG and SJ drafted the manuscript, while all authors proofread the article. All authors approved the final version of the manuscript.

\section{References}

1. Hankey GJ. Stroke. Lancet 2017; 389:641-54. https://doi. org/10.1016/S0140-6736(16)30962-X.

2. Favate AS, Younger DS. Epidemiology of ischemic stroke. Neurol Clin 2016; 34:967-80. https://doi.org/10.1016/j.ncl.2016.06.013.

3. World Health Rankings. Oman: Life Expectancy. From: www. worldlifeexpectancy.com/oman-life-expectancy Accessed: Sept 2021 .

4. Ng SW, Zaghloul S, Ali HI, Harrison G, Popkin BM. The prevalence and trends of overweight, obesity and nutrition区 related non-communicable diseases in the Arabian Gulf States. Obes Rev 2011; 12:1-13. https://doi.org/10.1111/j.1467-789X.20 10.00750.x.

5. Gujjar AR, Nandhagopal R, Ganguly SS, Shoaib R, Al-Asmi AR. Ischemic stroke outcomes in Oman: Experience of a university hospital based stroke registry. Neurol 2017; 88:278.

6. Deleu D, Inshasi J, Akhtar N, Ali J, Vurgese T, Ali S, et al. Risk factors, management and outcome of subtypes of ischemic stroke: A stroke registry from the Arabian Gulf. J Neurol Sci 2011; 300:142-7. https://doi.org/10.1016/j.jns.2010.08.023.

7. Wittenauer R, Smith L. Ischaemic and hemorrhagic stroke. From: www.who.int/medicines/areas/priority_medicines/BP6 6Stroke.pdf Accessed: Nov 2020.

8. Adams HP Jr, Bendixen BH, Kappelle LJ, Biller J, Love BB, Gordon DL, et al. Classification of subtypes of acute ischemic stroke. Definitions for use in a multicenter clinical trial. Stroke 1993; 24:35-41. https://doi.org/10.1161/01.str.24.1.35.

9. Kolominsky-Rabas PL, Weber M, Gefeller O, Neundoerfer B, Heuschmann PU. Epidemiology of ischemic stroke subtypes according to TOAST criteria: Incidence, recurrence, and long-term survival in ischemic stroke subtypes: a populationbased study. Stroke 2001; 32:2735-40. https://doi.org/10.1161/ hs1201.100209.
10. Zhou H, Li JJ, Wang YJ. [The reliability of ischemic stroke subtype classification using the TOAST criteria]. Zhonghua Nei Ke Za Zhi 2005; 44:825-7.

11. Schlesselman JJ. Case-Control Studies. Design, Conduct, Analysis. Oxford, UK: University Press, 1982

12. Ganguly SS, Al-Shafaee MA, Al-Maniri AA. Some risk factors for coronary heart disease among Omani males: A matched case-control study. Sultan Qaboos Univ Med J 2008; 8:45-51.

13. Al-Riyami A, Abdelaty MA, Jaju S, Morsi M, Al- Kharusi H, Al-Shekaili W. World Health Survey 2008. Muscat, Oman: Ministry of Health, 2012

14. Abboud H, Sissani L, Labreuche J, Arauz A, Bousser MG, Bryer A, et al. Specificities of ischemic stroke risk factors in Arabspeaking countries. Cerebrovasc Dis 2017; 43:169-77. https:// doi.org/10.1159/000454776

15. Hong KS, Lee J, Bae HJ, Lee JS, Kang DW, Yu KH, et al. Greater stroke severity predominates over all other factors for the worse outcome of cardioembolic stroke. J Stroke Cerebrovasc Dis 2013; 22:e373-80. https://doi.org/10.1016/j. jstrokecerebrovasdis.2013.04.008.

16. Stead LG, Gilmore RM, Bellolio MF, Jain A, Rabinstein AA, Decker WW, et al. Cardioembolic but not other stroke subtypes predict mortality independent of stroke severity at presentation. Stroke Res Treat 2011; 2011:281496. https://doi. org/10.4061/2011/281496.

17. GrauAJ,WeimarC,BuggleF,Heinrich A,GoertlerM,NeumaierS, et al. Risk factors, outcome, and treatment in subtypes of ischemic stroke: The German stroke data bank. Stroke 2001; 32:2559-66. https://doi.org/10.1161/hs1101.098524.

18. Arboix A, Alió J. Cardioembolic stroke: Clinical features, specific cardiac disorders and prognosis. Curr Cardiol Rev 2010; 6:150-61. https://doi.org/10.2174/157340310791658730.

19. World Health Organization. Chronic diseases and their common risk factorsFrom: http://www.who.int/chp/chronic disease_report/media/Factsheet1.pdf Accessed: Nov 2020.

20. Shah SM, Shah SM, Khan S, Rehman S, Khan Z, Ahmed W, et al. "Addressing the impact of stroke risk factors in a case control study in tertiary care hospitals": A case control study in tertiary care hospitals of Peshawar, Khyber Phukhtoonkhwa (KPK) Pakistan. BMC Res Notes 2013; 6:268. https://doi. org/10.1186/1756-0500-6-268.

21. Al-Lawati JA, Panduranga P, Al-Shaikh HA, Morsi M, Mohsin M, Khandekar RB, et al. Epidemiology of diabetes mellitus in Oman: Results from two decades of research. Sultan Qaboos Univ Med J 2015; 15:e226-33.

22. Tadmouri GO, Nair P, Obeid T, Al Ali MT, Al Khaja N, Hamamy HA. Consanguinity and reproductive health among Arabs. Reprod Health 2009; 6:17. https://doi.org/10.1186/17424755-6-17.

23. Islam MM. The practice of consanguineous marriage in Oman: Prevalence, trends and determinants. J Biosoc Sci 2012; 44:571-94. https://doi.org/10.1017/S0021932012000016.

24. Rahim HF, Sibai A, Khader Y, Hwalla N, Fadhil I, Alsiyabi H, et al. Non-communicable diseases in the Arab world. Lancet 2014; 383:356-67. https://doi.org/10.1016/S0140-6736(13)62383-1.

25. Meyer BF, Alsmadi O, Wakil S, Al-Rubeaan K. Genetics of type 2 diabetes in Arabs: What we know to date. Int J Diabetes Mellit 2009; 1:32-4. https://doi.org/10.1016/j.ijdm.2009.03.003. 